\title{
Robert ROCHEFORT, Le bon consommateur et le mauvais citoyen
}

Paris, O. Jacob, coll. Sciences humaines, 2007, 312 p.

\section{Stéphanie Hurez}

\section{(2) OpenEdition}

\section{Journals}

Édition électronique

URL : http://journals.openedition.org/questionsdecommunication/2566

DOI : 10.4000/questionsdecommunication.2566

ISSN : 2259-8901

Éditeur

Presses universitaires de Lorraine

Édition imprimée

Date de publication : 31 décembre 2007

Pagination : 441-442

ISBN : 978-2-86480-849-7

ISSN : 1633-5961

Référence électronique

Stéphanie Hurez, «Robert rochefort, Le bon consommateur et le mauvais citoyen », Questions de communication [En ligne], 12 | 2007, mis en ligne le 12 avril 2012, consulté le 08 avril 2021. URL : http:// journals.openedition.org/questionsdecommunication/2566; DOI : https://doi.org/10.4000/ questionsdecommunication.2566

Ce document a été généré automatiquement le 8 avril 2021.

Tous droits réservés 


\title{
Robert ROCHEFORT, Le bon consommateur et le mauvais citoyen
}

Paris, O. Jacob, coll. Sciences humaines, 2007, 312 p.

\author{
Stéphanie Hurez
}

\section{RÉFÉRENCE}

Robert ROCHEFORT, Le bon consommateur et le mauvais citoyen. Paris, O. Jacob, coll. Sciences humaines, 2007, $312 \mathrm{p}$.

1 À l'heure où le « consommer propre et responsable » est au cœur des débats politiques, Robert Rochefort s'insurge, dans son dernier opus au titre évocateur, sur les curieux paradoxes que révèlent nos modes de vie. Cinq secteurs emblématiques - les OGM, la grande distribution, la voiture, l'argent et le tourisme - sont passés au crible pour saisir « comment, [tout en évoluant synchroniquement], la consommation et la citoyenneté se relaient ou au contraire se distinguent » (p. 15) dans chacun des actes à vocation " consommatoire ». Si de nombreux sondages témoignent de l'engouement grandissant des consommateurs à devenir des citoyens engagés et éthiquement irréprochables, l'auteur ne cache pas son inquiétude face à la réalité de leurs agissements. Bien que sensible aux signes énonciateurs d'un changement qu'ils manifestent de manière ponctuelle - achat d'un produit issu du commerce équitable ou de l'agriculture biologique, par exemple -, il constate que rares sont ceux à satisfaire leurs besoins en fonction de ce qu'ils sont censés apporter aux autres, c'est-à-dire les considérer au-delà de leurs propres intérêts. Pour autant, il conçoit que «l'évocation de la citoyenneté dans la consommation n'est pas spontanée. C'est même a priori le contraire [car ces] deux notions ne relèvent pas des mêmes schémas mentaux. La consommation est immédiate et de plus en plus égocentrée. La citoyenneté est supposée réfléchie et renvoie à des responsabilités collectives »(p. 97). Difficile alors de les concilier au quotidien et, quand bien même y parviendraient-ils, leur liberté s'en trouverait plus ou moins affectée dans le sens où ils ne consommeraient plus seulement pour le plaisir- 
leur plaisir - mais pour contenter une nécessité sociale. Tout aussi légitime qu'elle soit, Robert Rochefort démontre, en évoquant le principe de précaution engendré par la vache folle et la grippe aviaire ou encore le réchauffement climatique qui ont contraint les consommateurs à changer radicalement leurs habitudes, qu'elle façonne leurs comportements en les rendant responsables de leur conduite, en les culpabilisant, ce qui prouve que le lien de cause à effet n'y est somme toute pas étranger.

2 Ironie du sort, les consommateurs n'auraient-il pas, de temps à autre, de bonnes raisons de se comporter en mauvais citoyens ou, plus encore, n'y seraient-ils pas inconsciemment encouragés? Autrement dit, est-ce qu'il est possible de demander à chaque individu de consommer sainement quand toutes les conditions ne sont pas réunies pour y parvenir? Ces questions complètent le raisonnement de l'auteur qui explique, à juste titre, que les personnes ayant un statut précaire ne peuvent pas s'adonner à une consommation citoyenne parce que leurs achats sont raisonnes en fonction de leurs ressources, mais ses analyses passent parfois à côté de certaines réalités où la pensée citoyenne n'a plus cours et qu'il aurait été bon de souligner La Logan de Renault en est une parfaite illustration : c'est une voiture « $\mathrm{I}^{\mathrm{er}}$ prix » qui, avant de conquérir les consommateurs européens, était destinée aux marchés émergeants (notamment l'Inde et la Russie), sauf que, à trop vouloir réduire les coûts de fabrication pour répondre aux contraintes économiques de ce segment, les modèles qui lui sont réservés ne répondent pas aux normes environnementales en vigueur sur le plan international - notamment en terme de pollution. C'est donc bien plus qu'un simple manque à gagner pour le marché de l'occasion français qu'elle pose. On comprend alors aisément que, au travers du spectre de la consommation idéalisée que promeuvent les discours moralisateurs mais légitimes des politiques et de tous ceux qui en défendent les valeurs, le consommateur ne soit pas toujours disposé à donner une portée citoyenne à chacun de ses actes. Nonobstant ce constat, Robert Rochefort poursuit sa réflexion en auscultant un secteur où la dimension éthique semble avoir, en apparence, plus de chance de gagner favorablement du terrain: celui du tourisme. En raison de leurs prix attractifs, les voyages en Tunisie et au Maroc sont, par exemple, victimes de leur succès. Face aux besoins apparents des habitants, les vacanciers qui sont un tant soit peu magnanimes ne peuvent qu'être compatissants et faire preuve de générosité sous quelque forme que ce soit. Cependant, il explique que si les initiatives se potentialisent et que le touriste «est prêt à faire des sacrifices pour se payer [du] dépaysement [...] il est rarement d'accord pour que cela se fasse au détriment de son confort de vie d'Occidental» et ne manque pas de préciser que «ce qui va rendre difficile le tourisme éthique dans les prochaines années résulte de cette concomitance difficile à gérer dans un secteur qui dans le même temps continue à se démocratiser, mais a besoin de modifier ses pratiques pour les rendre plus acceptables » (p. 244).

Concrètement, qu'en sera-t-il dans quelques années ? L'image du bon consommateur et $\mathrm{du}$ bon citoyen aujourd'hui peu engagé a-t-elle des chances de dépasser le stade de l'utopie en devenant une réalité, en faisant partie intégrante de notre quotidien? Ferme dans ses convictions, l'auteur estime qu'« un acte de consommation pourrait être "citoyen" dès lors qu'il ne vise pas seulement à la maximisation de l'intérêt individuel du consommateur, mais qu'il se préoccupe de l'intérêt général » (pp. 304-305), c'est-à-dire qu'il devienne la responsabilité de tous. Plus facile à dire qu'à faire ? Pas si sûr pour peu que, comme le soutient Robert Rochefort, l'instauration de la TVA sociale à l'horizon 2009 puisse marquer un premier pas dans l'économie solidaire. 
Mais la consommation sera-t-elle pour autant plus précautionneuse et toujours partisane d'une économie mondiale où l'altruisme a acquis certaines de ses lettres de noblesse ? Les débats étant d'ores et déjà ouverts, ils ne manqueront probablement pas de s'intensifier dans les prochains mois et ils permettront certainement à l'auteur d'affirmer ou d'infirmer avec plus de certitude la thèse qu'il défend dans cet ouvrage.

\section{AUTEURS}

\section{STÉPHANIE HUREZ}

CREM, université Paul Verlaine-Metz

stephanie.hurez@wanadoo.fr 